\title{
USES FOR EARTHQUAKE DETECTORS
}

\author{
R.G. Tyler* and D.E. Baguley*
}

\section{ABSTRACT:}

The history and use of earthquake detectors in New Zealand is described, both for switching on earthquake recorders and for switching off energy sources or equipment in the event of an earthquake. Their use as earthquake alarms, in particular to give a warning when a certain level of acceleration has been reached is discussed. For a warning device mounted in a structure suitable filtering is needed to ensure that the threshold acceleration is related to the response of the structure.

\section{INTRODUCTION :}

Much of the devastation caused by earthquakes in the past has arisen from the secondary effects of fire or explosion because of the failure to seal off fuel supplies or ignition sources on first arrival of an earthquake. Also lives may be lost by the failure of lifts between floors, when an early detection of the earthquake might have allowed passengers to leave the conveyance at the next floor. The pressure, or "P-waves" arrive before the destructive shear, or "s-waves", which allows a short delay for effective switching operations activated by the P-wave arrival, although the delay may be only of a few seconds for locations near the epicentre.

Up to about 1965 earthquake detectors were pendulum devices responding to horizontal ground motion which closed contacts when a certain amplitude of motion was reached. Other devices, used for switching off gas supplies, were based on the earthquake motion dislodging a ball horizontally from a seating to drop down to close a valve. Quite apart from the somewhat unreliable response of such devices, the advantage in time of picking up the earlier arrival of the $P-$ wave is lost, as it has been established worldwide that the P-wave causes primarily vertical motion. Hence a Pwave can be detected most reliably by a sensor for vertical motion.

In 1965, electromagnetic detectors, utilising a spring-mass system were introduced. When mounted vertically these allowed the P-wave to be detected and gave the earliest possible detection of the onset of an earthquake.

In addition to their use for switching devices, earthquake detectors are also used as warning devices. They may either be used to warn operators in noisy or critical industrial situations, such as nuclear power plants, that an earthquake is taking place, or more specifically that a particular value of acceleration has been reached in some part of the structure, when it may serve as a warning that overstressing has resulted.

*Physics and Engineering Laboratory, DSIR, Gracefield, Lower Hutt

\section{DETECTOR FOR MECHANICAL-OPTICAL (MO)} TYPE STRONG-MOTION EARTHOUAKE RECORDER:

An electromagnetic detector was designed in New Zealand in 1966 to trigger the newly developed mechanical-optical (M01) strong-motion recorder. It is still used in the M02 and M02A recorders which are deployed in the New Zealand recorder network. It is a velocity transducer, spring-mounted vertically with low damping and a natural frequency of about $6 \mathrm{~Hz}$ (Fig 1). The instrument is normally triggered when vertical excitation from the P-wave causes the peak output voltage from the coil to exceed the forward bias of two transistors connected in cascade.

Two detectors of this type were calibrated at PEL by detaching them from M02 recorders and mounting them on a vertical shaking table, driven sinusoidally at frequencies from $2 \mathrm{~Hz}$ to $50 \mathrm{~Hz}$, the accelerations being measured by an accelerometer. The results for the two coils were similar and results for one are reproduced in Fig. 2. The acceleration required to trigger the instrument normally employed in the field, which has a $200 \mathrm{k}$ damping resistor(1), is given by the full curve of Fig 2, for a steady sine wave input. The sensitivity may be reduced by decreasing the value of the resistor, as shown by the dotted curves in the same figure. For the normal value of damping resistor, and at resonance, the instrument is seen to trigger at about $0.001 \mathrm{~g}_{\mathrm{v}}$, where $g_{v}$ is the acceleration due to gravity $\left(9.81 \mathrm{~ms}^{-2}\right)$. However, an earthquake gives a complex input (2) and the instrument has been shown to have the required degree of sensitivity on the basis of operation in the New zealand strong-motion recorder network (3) during a period more than a decade. Earthquake records from the M.O. accelerographs show that the trigger level is about $0.01 \mathrm{~g}_{\mathrm{v}}$ for earthquake excitation.

The instrument has been used with reduced sensitivity in industrial situations, particularly in steel structures where vibration caused by machinery, or local impacts, causes so many triggerings that the instrument rapidly runs out of film. In these circumstances the most suitable value of damping resistor is found by trial and error. Ideally, however, detectors of any type should be mounted on a concrete plinth well away from factory noise, either in the foundations of the building or in a 


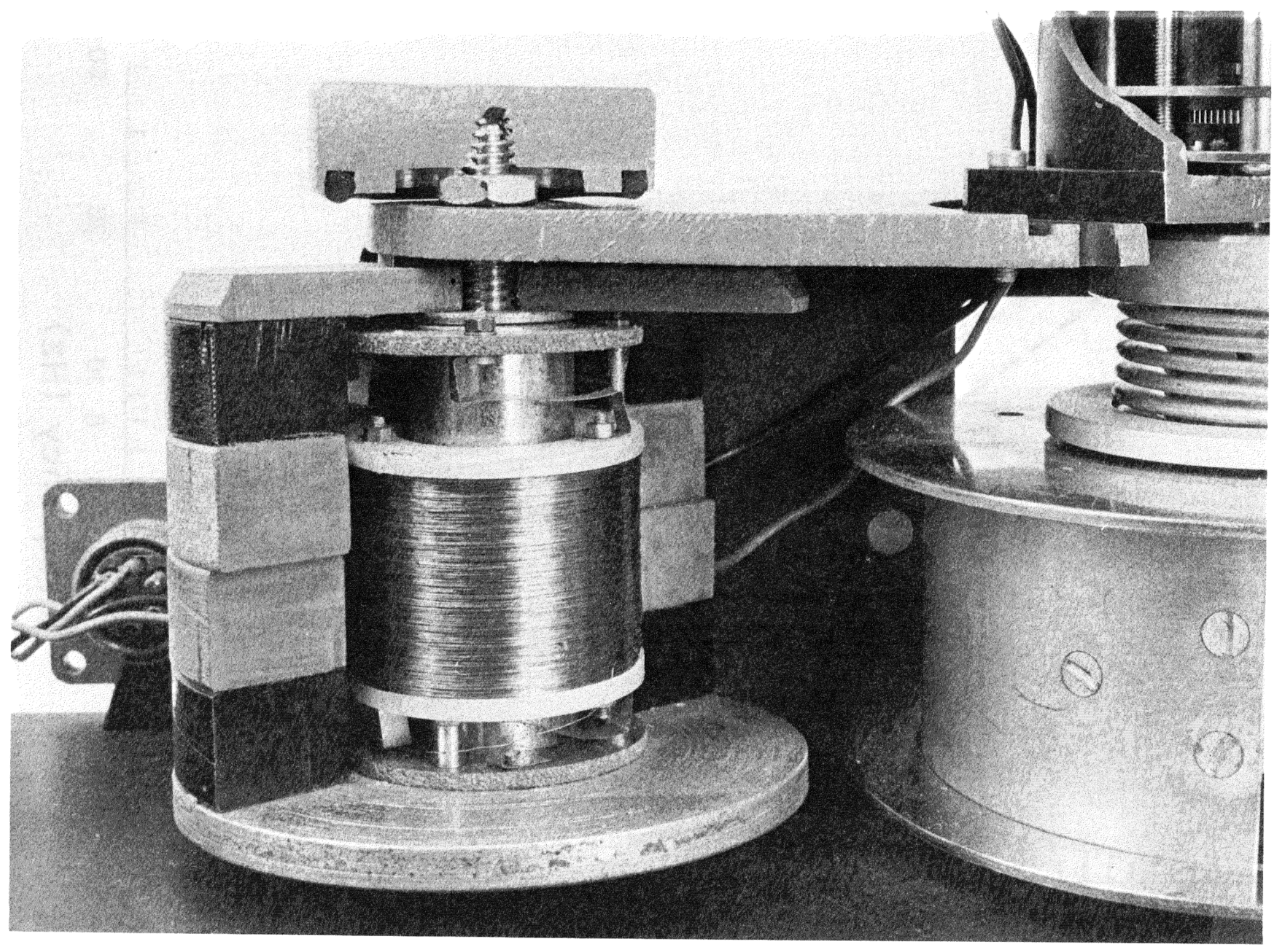

Figure 1. Cut-away view of coil unit for trigger in $\mathrm{MO} 2$ Strong Motion Recorder. 


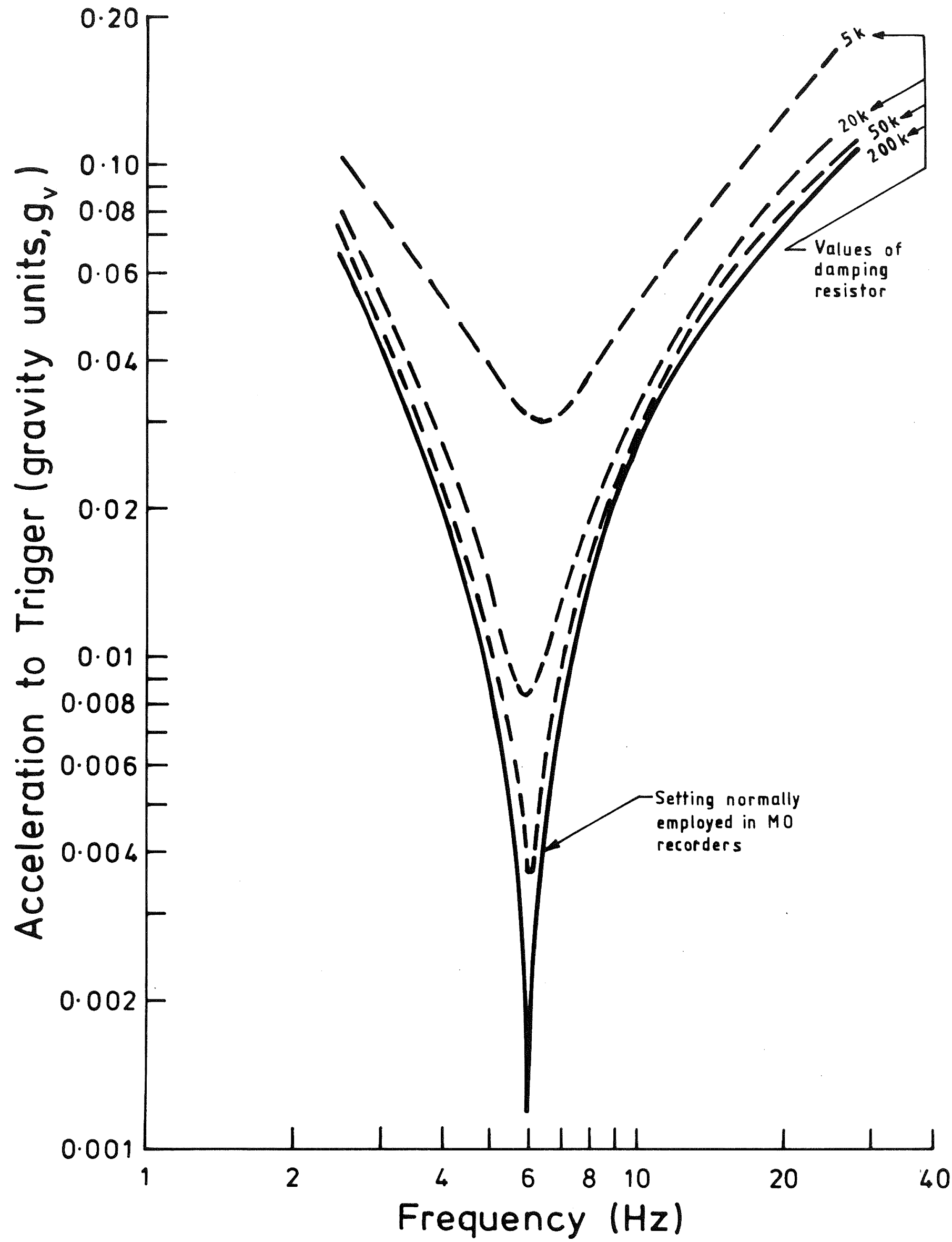

Figure 2. Characteristics of MO Type Detector 
separate shelter on the perimeter of the grounds, or in a waterproof shelter with a substantial cover box.

The MO type of detector uses a very small current input on standby. Power is required following triggering, to energise a relay. Power requirements thereafter depend on the type of switching operation to be carried out and should preferably be independent of mains supply. For the MO recorders a $12 \mathrm{~V}$ long-life dry-battery pack is used, with the capability of operating the recorder on triggering from a spontaneous start after 8 to 12 months on standby.

\section{SERVICE USE FOR DETECTORS:}

\section{(a) Switching mode}

The first detector employed to switch off equipment in the event of an earthquake was installed by PEL in the ICI Tasman Vaccine Works at Upper Hutt in 1968. It is used to submerge a radioactive source in water and was adapted from an MO recorder. It has functioned satisfactorily since installation and from time to time surprises nearby operators by submerging the source before anyone has noticed the arrival of the earthquake. The whole operation is powered by batteries and is therefore independent of mains supply.

A device of more recent design (Fig 3), manufactured by Solid State Equipment Ltd of Lower Hutt, employs a geophone with appropriate filter circuits to give the required frequency response. Normally the geophone is mounted vertically to pick up the $\mathrm{P}$-wave, but it can also be mounted horizontally, if required, when it will normally be employed in the warning mode (cf para $3(b)$ ).

The device is mains powered as there is a significant standby current drain. Auxiliary nickel-cadmium batteries, which are trickle charged, ensure operation for 40 hours in the event of a mains failure. The device, as normally manufactured, provides for triggering at three levels of vertical acceleration, $1 \%, 3 \%$ and $10 \% \mathrm{~g}_{\mathrm{v}}$ these settings can be varied to suit customer requirements. The roll-off at higher frequencies can also be adjusted to suit requirements, as it is desirable that high frequency vibration caused by knocks and bangs in the vicinity of the instrument do not cause triggering. The frequency response employed in earlier models is shown in Fig 4, curve 1 , established from tests carried out at PEI on a vertical shaking table (Fig 5), for frequencies between $2 \mathrm{~Hz}$ and $50 \mathrm{~Hz}$. The curve is shown for the $0.01 \mathrm{~g}$ setting but the shape is similar for the other settings. The characteristic shown in curve 2 , is proposed for detectors manufactured in 1981, to further cut the effects of higher frequencies. The characteristics for two detectors originating in the U.S.A. are also given in Fig $4(2)$.

In New Zealand, at the present time, most of the detectors in service are of the geophone type. Some are installed to switch off gas supplies; one is employed to bring the Wellington Cable car to an early stop. Other uses that could be included are:

Turning off flame sources

Turning off electric power to cables and lines

Switching on emergency power supplies in hospitals and essential service areas Switching on emergency lighting Bringing lifts to a stop on the next floor and opening the doors

Triggering a controlled power-down on computers

Automatic covering of vats of toxic or corrosive liquid to prevent splashing

In one unusual application for the New Zealand Electricity Department, an earthquake detector has been used to maintain equipment in operation rather than switch it off. This is in connection with Buchholz relays which are used to detect electrical breakdown in large transformers and hence to isolate a defective one. Because of their construction, the relays are sensitive to long-period excitations, and low-level shaking from distant earthquakes has switched off transformers unnecessarily. The earthquake detector shorts out the relays when an earthquake occurs so that the transformers are kept in operation. Its characteristic is similar to that of curve 2, Fig 4, with a roll-off at $12 \mathrm{~dB}$ per octave for frequencies above $10 \mathrm{~Hz}$.

The price for the detectors available commercially in New Zealand is about NZ\$600 in 1981.

\section{(b) Warning Mode}

In noisy industrial situations an earthquake detector may simply be used to confirm to operators that an earthquake of significance is taking place. For this application a ground acceleration of $0.1 g_{\mathrm{V}}$, detected in either of two detectors mounted orthogonally in a horizontal plane, is often a suitable threshold above which attention should be paid to the effects of the earthquake. Such a device could also be mounted remotely on an unattended dam or structure to telemeter in a warning to operators in a distant control station. More sophisticated devices may seek to give a red-light warning when a dangerous level of acceleration has been reached in a structure. This gives an immediate indication of response levels as an alternative to employing a strong-motion accelerograph and processing the record, which may take hours or days. For monitoring structural response a pair of orthogonally mounted detectors in the horizontal plane should be mounted in a critical position in the structure. Dangerous levels of acceleration are associated with large deflections arising from movement in the fundamental and the first few higher modes. Thus the detector should have a comparatively narrow bandwidth in its frequency response to include only the fundamental and first few modes of the structure. It is evident that a knowledge of the characteristics of the structure is required which can be obtained by a study of its ambient vibrations. 



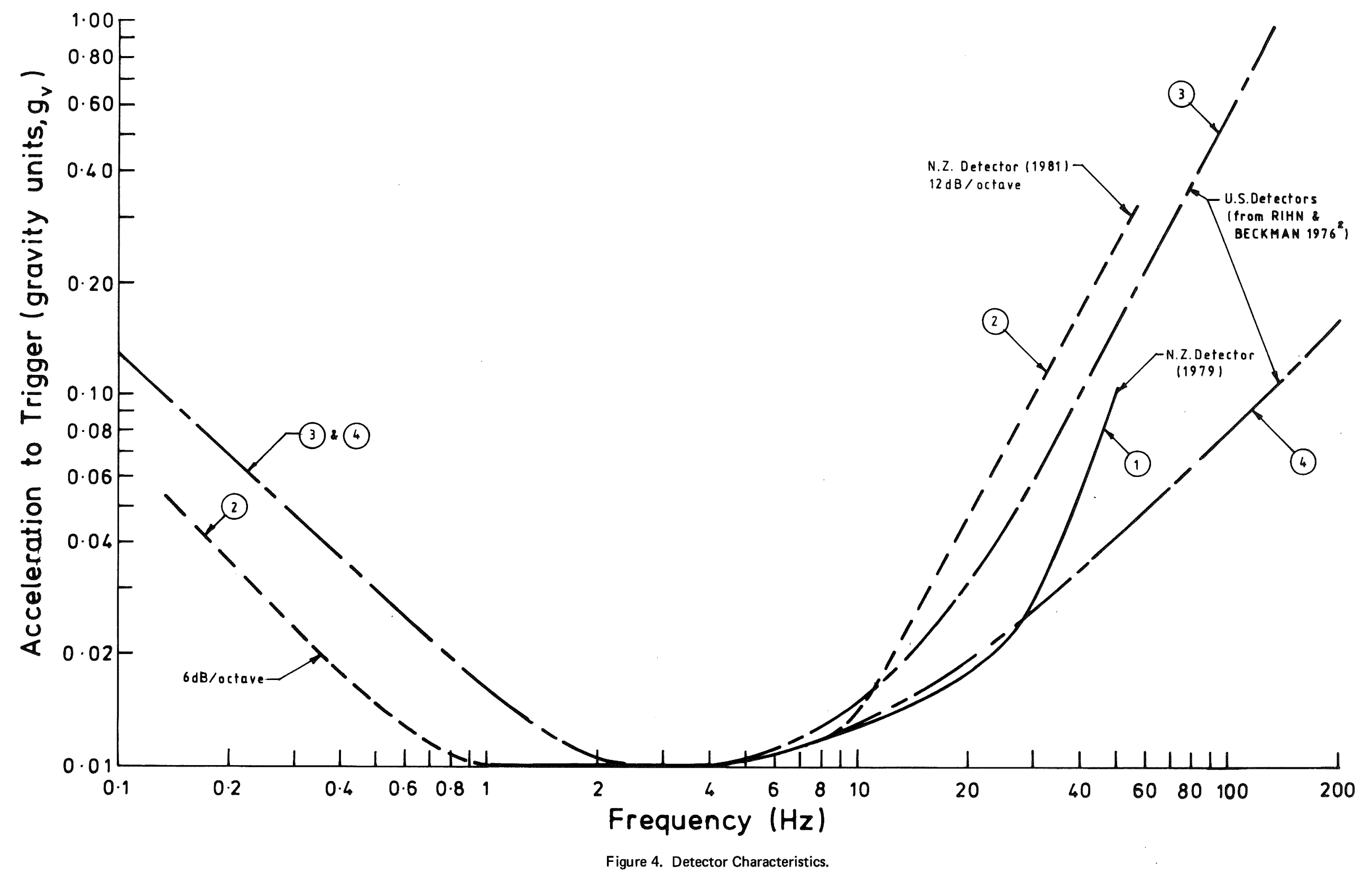


an off-shore platform having a fundamental frequency of $0.4 \mathrm{~Hz}$ is shown in Fig 6 . The bandwidth was 0.3 to $2.0 \mathrm{~Hz}$ and the design roll-off from $2 \mathrm{~Hz}$ was $24 \mathrm{~dB}$ per octave and alternative thresholds at $0.05 \mathrm{~g}_{\mathrm{V}}$, $0.10 \mathrm{gv}$ and $0.14 \mathrm{~g}_{\mathrm{v}}$ were provided.

Calibration was carried out for the lower frequencies using the pendulum shown in Fig 7, having a length variable up to about $2 \mathrm{~m}$; a vertical shaking table was used to establish the roll-off at higher frequencies. This could only be done for the $0.05 \mathrm{gv}$ curve because of lack of power in the available equipment but the shape of the curves for the higher thresholds of acceleration would be similar.

\section{ACKNOWLEDGEMENTS:}

Helpful discussions with W.R. Stephenson, originator of the detector employing a geophone, and with the staff of Solid State Equipment Ltd, are gratefully acknowledged; also the advice and comments of Dr R.I. Skinner, originator of the MO type earthquake recorder, and of $\mathrm{Dr} \mathrm{J} . \mathrm{L}$. Beck, are appreciated.

\section{REFERENCES:}

1. Skinner-Douflou, "Type MO2 Accelerograph Installation and operating Manual". Victoria Engineering, Lower Hutt, New Zealand.

2. Rihn, W.J. and W.J. Beckmann,

"Characteristics of Electromagnetic Starters for Strong-Motion Accelerographs". Bull. Seis. Soc. Am. 67. 6, 1667-1669, Dec. 1977.

3. Hefford, R.T. et. al, "The New Zealand Strong-Motion Recorder Network", Bull. N.z. Bat. Soc. Earthq. Eng. 12, 3, 256-263, Sept. 1979.

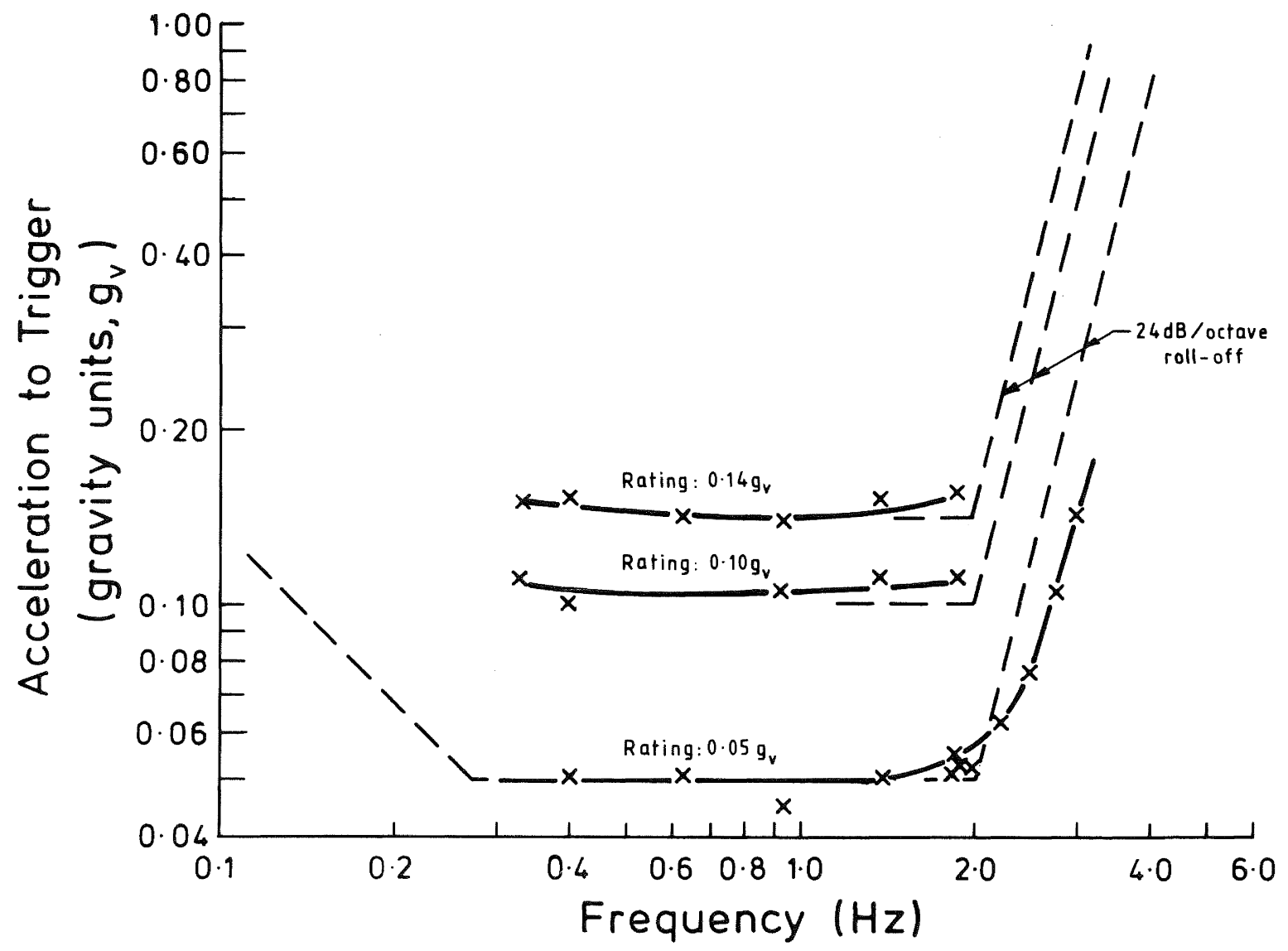

Figure 6. Characteristics of Earthquake Warning Device. (dotted curves give required design characteristics) 


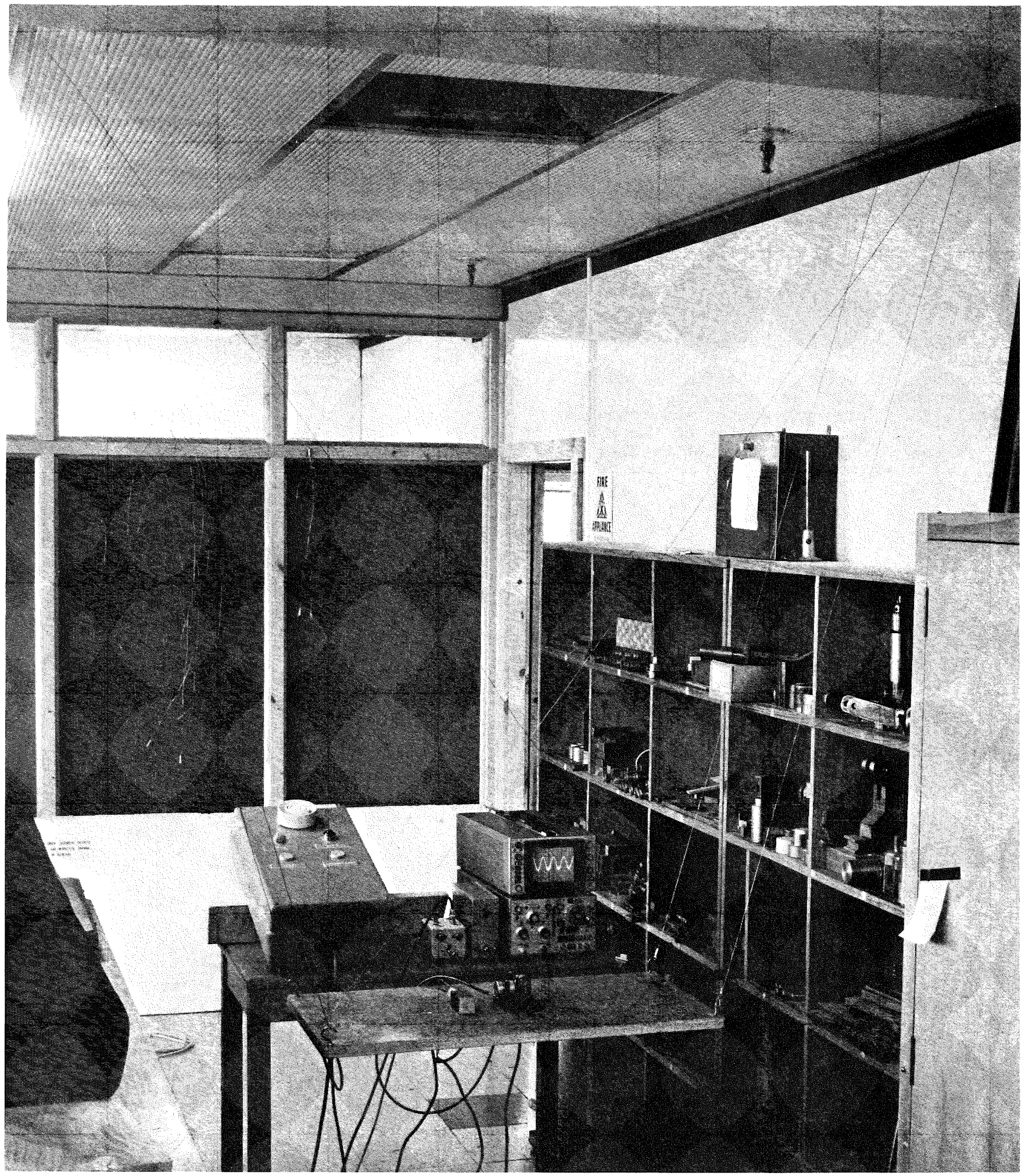

Figure 7. Platform pendulum, up to $3 \mathrm{~m}$ long, suspended from the ceiling by wires to enable sensors for warning devices to be calibrated at low frequencies. 